\title{
URBAN TREES IN UNIVERSITY CAMPUS: STRUCTURE, FUNCTION, AND ECOLOGICAL VALUES
}

\author{
Wang Xueyan (王雪岩) \\ 沈阳工学院 (Shenyang Institute of Technology)
}

\begin{abstract}
: university campuses usually have more trees and can provide various ecosystem services. However, there are few reports on tree ecosystem services of Chinese university campuses, especially in northern China. This study investigated the trees in the campus of Shenyang Institute of Technology and analyzed its ecological benefits and monetary value through i-Tree Streets. The campus trees contained a total of 5193 trees of 66 species, of which Catalpa ovata G. Don, Acer mono Maxim., Rhus Typhina Nutt, and Salix babylonica L. accounted for 59.7\% of the total number. The age structure of the trees in the campus was not ideal, with $71.5 \%$ of young trees, $24.0 \%$ of maturing trees, $4.5 \%$ of mature trees, and only $0.04 \%$ of old trees. The trees in campus provided more energy savings benefits (\$60,850, \$11.7/tree), carbon reduction benefits $(\$ 34,318, \$ 6.6 /$ tree) and aesthetic benefits $(\$ 30,150$, $\$ 5.8 /$ tree). The benefits resulted from air pollutants removal $(\$ 12,889, \$ 2.5 /$ tree) and rainwater runoff interception (\$15,534, \$3.0/tree) were smaller. In addition, tree species with more maturing trees and mature trees (i.e., with larger diameter at breast height) and large leaf area in the campus contributed significantly to ecosystem services. Our results can provide suggestions and certain insights for Chinese campus greening managers in tree species selection and tree management.
\end{abstract}

Keywords: University campus, tree, i-Tree Streets, ecosystem service.

Rapid urbanization has disturbed the natural urban ecosystem and degraded the urban environment. Campus trees, as a part of urban forests, can not only increase the plant coverage in the campus, maintain fresh air, improve the microclimate and campus ecological indicators, but also maintain the physical and mental health of teachers and students. However, previous studies mainly focused on the ecological benefits of street trees, urban parks, and residential areas, etc., only few studies existed on campus trees and focus on the single function of campus trees. Here, we first applied the i-Tree Streets to study the structure and benefits of trees in Shenyang Institute of Technology (SIT), Fushun, China. Our main objectives include: (1) analyzing the tree structure in the campus of Shenyang Institute of Technology; (2) quantifying its ecosystem services in monetary terms, including energy savings, carbon reduction, air pollutants removal, rainwater runoff interception and aesthetic benefits. We hope to provide a certain basis for the management of urban trees in the campus through the investigation and research on the trees in the campus of Shenyang Institute of Technology.

We found that campus trees were estimated to be 5,193 trees and 66 species have been identified. However, the campus relies too much on C. ovata and S. babylonica, easily generating serious management concerns. In addition, the age structure of campus trees was not ideal, with a large proportion of young trees $(71.5 \%)$, fewer maturing and mature trees $(28.5 \%)$ and a deficit of old trees $(0.04 \%)$, suggesting that campus trees were the "growth type" and will provide more ecosystem benefits in the future. By using i-Tree Streets, the total benefits produced by campus trees were valued at $\$ 153,766$ ( $\$ 29.6 /$ tree). Among the total benefits, energy savings benefits contributed the most, accounting for $39.6 \%$, followed by carbon reduction benefits $(22.3 \%)$ and aesthetic benefits (19.6\%). Smaller benefits resulted from air pollutants removal (8.4\%) and rainwater runoff interception (10.1\%). Among the predominant tree species, S. babylonica and R. pseudoacacia contributed more to the total benefits due to more maturing and mature trees and therefore large leaf area, which was consistent with previous studies that trees with larger leaf area can contribute more benefits of ecosystem services. In contrast, for A. pseudosieboldianum dominated by young trees, although the number of trees ranks tenth $(1.8 \%)$ among all campus trees, their contribution to the benefits of ecosystem services was scarce. In general, campus trees of SIT were in a sustainable and healthy state, which can provide more benefits in the future. However, campus greening managers should consider the ideal proportion of tree species and to plant trees with larger DBH and larger leaf area. 